\title{
AESTHETICS, DYNAMICS, AND MUSICAL SCALES: A GOLDEN CONNECTION
}

\author{
Julyan H. E. Cartwright ${ }^{*}$, Diego L. González ${ }^{\dagger}$, Oreste Piro $^{\S}$, \& Domenico Stanzial ${ }^{\dagger}$ \\ ${ }^{*}$ Laboratorio de Estudios Cristalográficos, CSIC, E-18071 Granada, Spain \\ ${ }^{\dagger}$ Laboratorio di Acustica Musicale e Architettonica, CNR - Scuola di San Giorgio, \\ I-30124 Venezia, Italy \\ ${ }^{\ddagger}$ Istituto Lamel, CNR, I-40129 Bologna, Italy \\ §Institut Mediterrani d'Estudis Avançats, CSIC - Universitat de les Illes Balears, \\ E-07071 Palma de Mallorca, Spain
}

Published in J. New Music Research 31, 51-58, 2002.

\begin{abstract}
Number theory has recently found a quantity of applications in the natural and applied sciences, and in particular in the study of nonlinear dynamical systems. As our sensory systems are highly nonlinear, it is natural to suppose that number theory also plays an important rôle in the description of perception, including aesthetics. Here we present a mathematical construction, based on number-theoretical properties of the golden mean, that generates meaningful musical scales of different numbers of notes. We demonstrate that these numbers coincide with the number of notes that an equal-tempered scale must have in order to optimize its approximation to the currently used harmonic musical intervals. Scales with particular harmonic properties and with more notes than the twelve-note scale now used in Western music can be generated. These scales offer interesting new possibilities for artists in the emerging musical world of microtonality and may be rooted in objective phenomena taking place in the nonlinearities of our perceptual and nervous systems.
\end{abstract}

\section{Introduction}

From antiquity humanity has sought through scientific enquiry a rational explanation of nature. As artworks were considered an imitation of nature, the same purpose has pervaded the history of the arts. The Pythagoreans were the first to put into mathematical terms the rules for aesthetics, borrowing them from music (Allott, 1994). Later there arose the concepts of eurhythmy or commodulation: the application of rhythmical movements or harmonious proportions in a piece of music; a painting; a sculpture; a building; a dance. Throughout the Middle Ages, mathematical ideas of proportion lived side by side with the body of artistic activity, but during the Renaissance, the natural sciences and mathematics began a process of separation from the arts, both theoretically as well as in practical terms (James, 1993). One of the reasons for the divorce was that all efforts failed to give a rational basis to the rôle played by numerical proportions in the aesthetics of an artwork. This lack of scientific rationale caused a rejection of works on numerical proportion in aesthetics by the scientific community, which began to consider writings in this area esoteric and unscientific. The divergence between arts and sciences grew wider in the twentieth century, with the end of the last movements retaining the ancient mathematical roots of art: neoclassicism and cubism. From this point on, the tendency of artists has been to consider that the mathematical design of an artwork implies an unacceptable constraint to creativity. If, in the future, the gulf between arts and sciences is to be reduced, this may come about through being able to understand in an objective fashion the phenomena that take place in our perceptual and nervous systems when we look at a painting (Zeki, 1999), or listen to music. Some of these phenomena may be rooted in the fundamental rôle in the theory of nonlinear dynamical systems played by a particular number: the golden mean. 


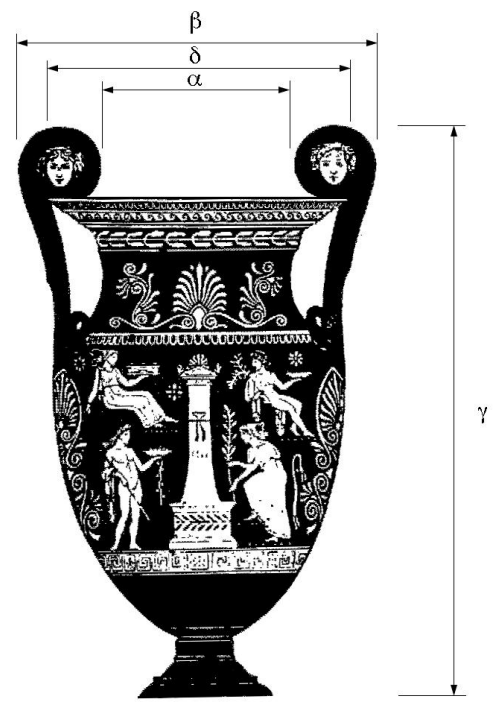

Figure 1: An example of golden number composition in a Greek amphora (4th century BC). The ratio between the greater and lesser diameters equals $\Phi: \delta / \alpha=1.618 \ldots$. The white line passing through the base of the handles also divides the total height $\gamma$ in the proportion $\Phi$. The use of the convergents of the golden number is also evident in the figure. For example, the height of the base of the handles is in 1:1 proportion - the first convergent of $\Phi$ - with the width $\beta$, determining the division of the golden rectangle $(\beta, \gamma)$ into a lesser golden rectangle by means of its gnomon: a square. Moreover, the horizontal line in the central column divides the total height in two equal parts, determining a ratio 1:2, the second convergent to $\Phi$. Finally, the line passing by the two small holes near the base of the handles determines a ratio of 2:3, the third convergent to the golden mean.

\section{The golden mean in art and science}

There exist many scientific, technical, and even esoteric writings about the use of the golden section, $\Phi=$ $(1+\sqrt{5}) / 2=1.618 \ldots$, and its companion $\phi=1 / \Phi=\Phi-1=0.618 \ldots$ in art (Ghyka, 1977; Huntley, 1970). There also exists a similar tradition regarding its rôle in science and technology (Schroeder, 1990, 1992). The number and some of its numerical properties were certainly known to the Greeks (Herz-Fischler, 1998), and it was possibly the key to the Pythagorean discovery of irrational numbers through its geometrical application to the pentagram. Platonic geometers named it 'the section' referring to its unique properties. Kepler described $\Phi$ as one of the 'jewels of geometry', but the name of golden mean, golden section, or golden number may first have been ascribed to it by Leonardo da Vinci. Da Vinci himself illustrated the book about $\Phi$ by Luca Pacioli, with whom da Vinci studied mathematics, which Pacioli entitled De Divina Proportione (Pacioli, 2001).

Whatever the level of theoretical knowledge about $\Phi$ and its attributed mystical significance, its use in painting, sculpture and architecture is certainly very ancient. Outstanding architectural examples are the Great Pyramid of Cheops and the Parthenon of Athens. Many ancient Chinese paintings also show the golden section, and achieve an accuracy of three decimal places in the major to minor ratio of some of their frames. Much Greek pottery also exhibits the golden section in the proportions of its parts. One example will suffice: in Fig. 1 we show a sketch of a Greek amphora of the 4th century BC. The ratio of the major to the minor diameter of the amphora equals $\Phi$ to three decimal places; the golden section is present also in many other details of the object, for example, the total height is divided in the proportion $\Phi$ by the white horizontal line at the base of the handles.

A number of hypotheses have been put forward as explanations for the constant presence of $\Phi$ in art. The most 


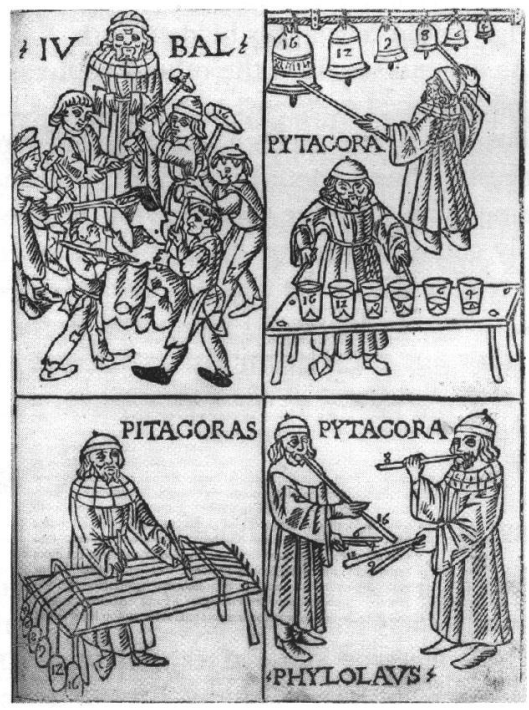

Figure 2: Woodcut by Franchino Gafurio, music theorist and choirmaster of Milan cathedral, shows at upper left the legendary discovery of the harmonic intervals by Pythagoras in a smithy. Pythagoras is also shown investigating with bells, strings and flutes. [Frontispiece of F. Gafurio "Theorica Musice" Naples (1480)].

accepted refers to a cultural fact: because the golden proportion is found frequently in nature - in phyllotaxis, sea shells, seed heads, human proportions, etc. — and because art was considered originally an imitation of nature, it is natural to find this proportion in different artworks. This explanation, however, begs the question: why is the golden section ubiquitous in nature? The ancient Greeks argued that the explanation for this ubiquity of $\Phi$ must be sought in a mathematical description of the world, and that numbers - the branch of mathematics now known as number theory - describe all things in the universe. They developed a theory of proportions as an explanation for our aesthetic perception of the universe and as a guide for the work of artists. A proportion is the equality of at least two ratios: $r=a / b=c / d$. This is termed a discrete proportion because the four elements are distinct. If two elements of the proportion coincide, the proportion becomes continuous. For example, if $b=c$, the proportion reads $r=a / b=b / d$, which has the solution $b=\sqrt{a d}, r=\sqrt{a / d}$, when $b$ is known as the geometric mean of $a$ and $d$. We can further simplify the proportion by making one element dependent on the other two. Given $d=a+b$, so the ratio of the smaller part $a$ to the larger part $b$ is the same as the ratio of the larger part $b$ to the whole $a+b$, we obtain only two possibilities for $r: \phi=(\sqrt{5}-1) / 2=0.618 \ldots$, and $-\Phi=-(1+\sqrt{5}) / 2=-1.618 \ldots$; this is the geometric definition of the golden section.

In art, the appropriate links between proportions of the parts and the whole gives to the artwork the quality of eurhythmy. Eurhythmy is currently more generally associated with arts that work in the time dimension, such as music or dance, but in antiquity it was used equally for the arts working with the spatial dimensions, such as painting, sculpture or architecture. Many artists have attempted to develop a parallelism between figurative and non-figurative arts; the writing of da Vinci on music and painting is famous. We can find such projects in modern painting also. Gino Severini, for example, tried to put musical rules into visual terms, while Paul Klee held, as did Goethe, that colour may be managed through a general theory of composition in the same way that sound is managed through the framework of musical theory: a sort of synthesis like that obtained in the works of Bach or Mozart. Less clear, however, is the contrary: the translation of the aesthetic rôle played by the golden number in painting, sculpture, and architecture to the musical world (see, for example, Huntley (1970); Lendvai (1966)).

Western science was born with the Pythagoreans, who developed the first mathematical model of a physical problem. This starting point also coincides with the start of rational studies of music, because the Pythagoreans 
Table 1: Names and frequency ratios of the currently accepted harmonic intervals in Western music in descending order of consonance.

\begin{tabular}{lll}
\hline Unison & Invariant & $1 / 1$ \\
Octave & Invariant & $1 / 2$ \\
Fifth & Invariant & $2 / 3$ \\
Fourth & Mixed & $3 / 4$ \\
\hline
\end{tabular}

\begin{tabular}{lll}
\hline Major Sixth & Variable & $3 / 5$ \\
Major Third & Variable & $4 / 5$ \\
Minor Third & Variable & $5 / 6$ \\
Minor Sixth & Variable & $5 / 8$ \\
\hline
\end{tabular}

developed a musical theory: that of harmonic musical intervals. Legend tells how Pythagoras entered a smithy and heard the noise of hammers of different masses working a great piece of incandescent iron. Some of the hammers striking simultaneously produced harmonious sounds. This motivated Pythagoras to study musical harmony with different tuneable instruments, as the medieval woodcut of Fig. 2 shows. In this way he identified at least the principal harmonic musical intervals: the unison, the octave, the perfect fifth and the fourth. His principal observation was that some simple numerical relationships defined these intervals (see Table 1 for the list of harmonic intervals currently accepted in Western music). Of course these numbers depend on the physical variables chosen to represent the sounds, but in time it emerged that the fundamental magnitude related to harmony is frequency. Fortunately, many numerological approaches maintain their validity because they work with the lengths of strings, since ratios obtained with these lengths are just the inverse of frequency ratios (a string fixed at both ends oscillates at a fundamental frequency proportional to the inverse of its length). Pythagorean ratios were quickly utilized for the construction of a musical theory. This musical theory was based fundamentally on the construction of a musical scale: the Pythagorean musical scale.

\section{The need for musical scales}

As a first approximation we can say that any frequency can be assigned a pitch, that is, a comparative sensation that allows us to say that a sound is higher or deeper than another. However, because there is a continuum of frequencies in any finite interval, there is an infinity of possible pitches. We should point out a couple of caveats: first, pitch can be ascribed directly to frequency only for pure tones (sounds that contain only one frequency in their spectrum), and for a definite intensity; second, the ear does not have an infinite resolving power, and thus two pure tones sufficiently close in their frequencies are judged to be of the same pitch. However, the resolving power is sufficiently high to be considered a continuum for the frequency values of the notes in any practical musical scale. For example, a semitone is given by a distance of 100 cents in the equal-tempered scale of twelve notes - there being 1200 cents in an octave — but the ear can distinguish a substantially lesser interval: the just noticeable difference limen is as little as three to four cents at $1000 \mathrm{~Hz}$. What then is the need for musical scales? A practical demonstration cannot give us the complete answer but can convince us of the practical necessity of a discretization of the octave into notes. If we take a known melody and replace the interval between notes by a continuum glissando the melody loses all its musical attractiveness and can become unidentifiable, despite the existence of the fixed frequency clues of the limit of the original intervals. This problem has long been recognized in practical terms and also, by the Pythagorean school at least, in theoretical terms.

The Pythagorean scale can be obtained by successive applications, ascending or descending from a tonic, of the interval of the perfect fifth. The notes obtained in this way must be replaced by their octave equivalents in order to have all the notes in the same octave. The Pythagorean process, however, has a problem because it never ends: an integer number of fifths never coincides with any other integer number of octaves; in numbertheoretical terms, the problem is that $2^{x}=3^{y}$ has no solutions if $x$ and $y$ are integers. The essence of the Pythagorean scale is the preservation of harmonic intervals, mainly the fifth and the octave. From Pythagoras up to the present day, many musical scales have been developed that try to accommodate the desire for harmonic intervals with the reality that they do not fit within the octave, the most important being the equal-tempered scale of twelve notes. Equal-tempered scales are defined by irrational numbers, and do not exactly preserve any of the harmonic intervals of Table 1 except for the octave, but, for some particular number of notes, they approximate 


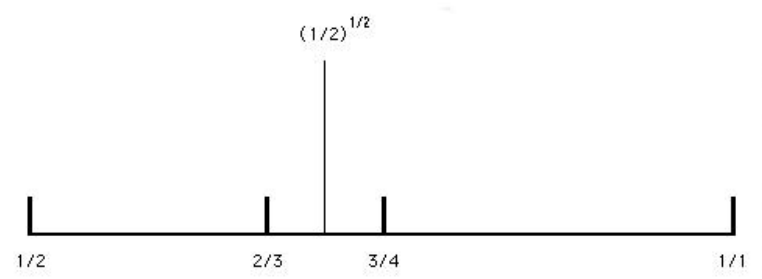

Figure 3: The octave interval, defined by the notes of frequency $1 / 2$, the tonic, and $1 / 1$, its superior octave, is divided by its geometric mean $\sqrt{1 / 2}$ as shown. The interval is defined by the first two convergents of the golden number, $1 / 1$ and 1/2, to which we have added the next convergent, 2/3. However, this breaks the symmetry of the scale. There exists another solution which consists of the permutation of the short and long intervals defined by $2 / 3$, i.e. $3 / 4$. This solution can be viewed as that symmetric to $2 / 3$ through the symmetry axis $\sqrt{1 / 2}$. Symmetry is meant here in the Greek sense, that is, as an equality of ratios, i.e. $(2 / 3) / \sqrt{1 / 2}=\sqrt{1 / 2} /(3 / 4)$. If we take logarithms of all quantities the symmetry becomes the usual sort and the geometric mean, $\sqrt{1 / 2}$, can be viewed as a mirror.

them.

\section{The golden scales}

The construction of a musical scale is then a problem involving approximating irrational numbers by rationals. The mathematical technique to obtain the best such approximations is well known, and consists of writing the irrational number as a continued fraction (Hardy \& Wright, 1975). The golden mean $\phi$ has the continuedfraction expansion

$$
\phi=\frac{\sqrt{5}-1}{2}=\frac{1}{1+\frac{1}{1+\frac{1}{1+\ldots}}},
$$

and the best rational approximations to $\phi$ are given by the convergents of this infinite continued fraction, arrived at by cutting it off at different levels in the expansion: $1 / 1,1 / 2,2 / 3,3 / 5,5 / 8,8 / 13$, and so on; the convergents of the golden mean are ratios of successive Fibonacci numbers.

Most musical scales are discretizations of the octave. The octave interval is such that the sensations produced by two notes separated by an octave are very similar, and harmonious when sounded simultaneously. This is independent of cultural roots or specific musical training, and is a shared characteristic that seems to be linked to human physiology. As the octave is an interval defined by the first and second convergents $1 / 1$ and $1 / 2$ of the golden number, we can attempt to construct a scale by continuing the series, adding the succeeding convergent of the golden mean $2 / 3$. The choice of a note $x$ in the octave interval $(1 / 2,1)$ satisfies the minimal condition to have a proportion: we have three elements $(1 / 2, x, 1)$ that define two ratios, $a=1 /(2 x)$ and $b=x$. However, the introduction of this rational number, $2 / 3$, breaks the symmetry of the interval because there are now two ratios defined, $a=(1 / 2) /(2 / 3)=3 / 4$ and $b=(2 / 3) / 1=2 / 3$. This is to say that there is a hidden solution that corresponds to the permutation of the intervals. If we equate the two ratios, $a=b$, this gives for $x$ the geometric mean of 1/2 and 1: $x=\sqrt{1 / 2}$. For the geometric mean the two ratios are equal; for the rational $2 / 3$ there is one interval greater than the other and the permutation corresponds to the exchange of these. If we include this hidden solution, $3 / 4$, we reestablish the symmetry as if a mirror were placed at the geometric mean (see Fig. 3). This palindromic character for a musical scale was first proposed by Newton in his notebooks written between 1664 and 1666. Newton pursued this idea further and presented in Opticks (Newton, 1952) the visible optical spectrum divided into ratios corresponding to those of a musical scale, with the divisions in the form of a palindrome. 

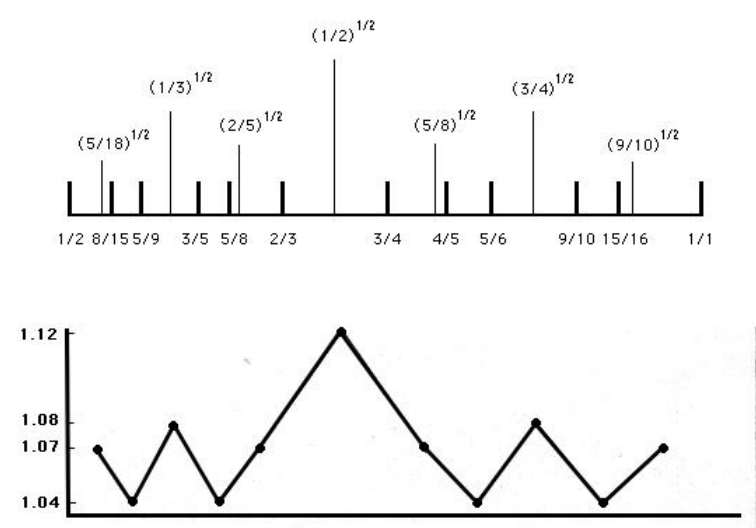

(a)

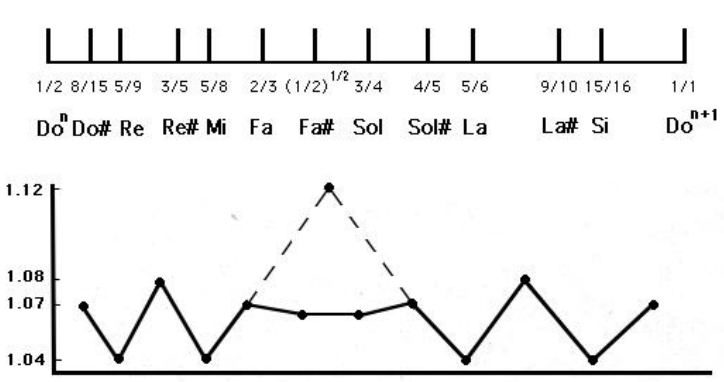

(b)

Figure 4: (a) The golden scale construction developed until the fifth convergent $c_{5}=5 / 8$ (upper panel), and the intervals between adjacent notes (lower panel). We can see that the intervals are distributed in a band. If we take as a rule that the intervals cannot be greater than the quotient of convergents $c_{n-2} / c_{n+1}$, in this case $c_{3} / c_{6}=(2 / 3) /(8 / 13)=13 / 12=1.08$, or less than that of the convergents $c_{n} / c_{n-1}$, here $c_{5} / c_{4}=(5 / 8) /(3 / 5)=25 / 24=1.04$, we find that the anomalous interval 1.13 , between $2 / 3$ and $3 / 4$, must be subdivided once. However there is no solution to this problem in rational numbers, because the inclusion of a rational number and its image generates at least one interval less than 1.04. The only possibility is thus to include the irrational axis $\sqrt{1 / 2}$ itself. (b) The result of including the irrational axis. We can see that all the intervals are now within the previously defined band. As the number of notes coincides with the number of notes of the usual equal-tempered scale of twelve notes, we have given the same names to the notes of this golden scale.

At this point we can generalize our procedure. For this it is sufficient to notice that the first note included, the new rational approximant to the golden mean, creates a new interval, $(1 / 2,2 / 3)$, which, as before, can be divided by a geometric mean which, in turn, can be approximated by a rational that corresponds to the succeeding approximant of the golden mean. This choice breaks the symmetry, which can be reestablished through the image of this approximant in the geometric mean mirror of this interval $\sqrt{1 / 3}$, and further, its image in the previous mirror $\sqrt{1 / 2}$. At the next level — including the convergent $3 / 5$ - this construction gives us a pentatonic golden scale; C (1/2), $\mathrm{D}^{\sharp}(3 / 5), \mathrm{F}(2 / 3), \mathrm{G}(3 / 4), \mathrm{A}(5 / 6)$, and C (1/1).

Now we need only a rule for proceeding in the subdivision of the interval: the maximum and minimum values for the intervals between successive notes. We can see in Fig. 4a, which shows the procedure performed until the third level (including the convergent 5/8), that all the intervals except one fall in a band determined by the ratios between convergents of the golden number. The greatest interval is that including the first geometric mean $\sqrt{1 / 2}$. If we seek to subdivide this interval further we find that there is no rational solution that preserves the palindromy inside the band. Thus the unique possible choice is the irrational geometric mean itself. This is curious, because we are forced to choose a note that is essentially different to the others, having an irrational interval. The scale at which we have arrived consists of twelve notes; the same number of notes as has the equaltempered scale now in use (see Fig. 4b). Moreover, the golden scale construction has generated all the harmonic intervals currently accepted by Western music (Table 1). Because of the equal numbers of notes, we can give the same names to the golden scale notes as their equal-tempered counterparts and compare their dispersion; see Table 2. It is intriguing that the irrational note corresponds to the interval $\mathrm{C}$ to $\mathrm{F}^{\sharp}$, which has long been a problem in musical theory because of its ambiguity: being difficult to define as consonant or dissonant. Because of this it has been named the 'diabolus in musica'; in our construction it is certainly an irrational devil!

In Fig. 5 we have calculated the mean quadratic dispersion as a function of the number of notes for an arbitrary equal-tempered scale. This is an indication of how well the harmonic intervals listed in Table 1 are simultane- 
Table 2: Comparison of the notes of the twelve-note equal-tempered scale with those of the golden scale with the same number of notes.

\begin{tabular}{llrrrr}
\hline \multicolumn{1}{c}{ Note } & $\begin{array}{r}\text { Equal-Tempered } \\
\text { Scale (Hz) }\end{array}$ & $\begin{array}{r}\text { Twelve-Note } \\
\text { Golden Scale (Hz) }\end{array}$ & $\begin{array}{r}\text { Difference } \\
(\%)\end{array}$ & $\begin{array}{r}\text { Difference } \\
(\text { cents })\end{array}$ \\
\hline $\mathrm{C}_{8}$ & $\mathrm{Do}_{8}$ & 4186.00 & 4186.00 & 0.00 & 0.0 \\
$\mathrm{C}^{\sharp}$ & $\mathrm{Do}^{\sharp}$ & 4434.92 & 4465.07 & 0.68 & -11.7 \\
$\mathrm{D}$ & $\mathrm{Re}$ & 4698.64 & 4651.11 & -1.01 & 17.6 \\
$\mathrm{D}^{\sharp}$ & $\mathrm{Re}^{\sharp}$ & 4978.03 & 5023.20 & 0.90 & -15.6 \\
$\mathrm{E}$ & $\mathrm{Mi}$ & 5274.04 & 5232.50 & -0.78 & 13.7 \\
$\mathrm{~F}$ & $\mathrm{Fa}$ & 5587.65 & 5583.33 & -0.11 & 2.0 \\
$\mathrm{~F}^{\sharp}$ & $\mathrm{Fa}$ & 5919.90 & 5919.90 & 0.00 & 0.0 \\
$\mathrm{G}$ & $\mathrm{Sol}^{\sharp}$ & 6270.96 & 6279.00 & 0.12 & -2.0 \\
$\mathrm{G}^{\sharp}$ & $\mathrm{Sol}^{\sharp}$ & 6644.87 & 6697.60 & 0.79 & -13.7 \\
$\mathrm{~A}$ & $\mathrm{La}^{\sharp}$ & 7040.00 & 6976.67 & -0.89 & 15.6 \\
$\mathrm{~A}^{\sharp}$ & $\mathrm{La}^{\sharp}$ & 7458.62 & 7534.80 & 1.02 & -17.6 \\
$\mathrm{~B}$ & $\mathrm{Si}$ & 7902.13 & 7848.75 & -0.68 & 11.7 \\
$\mathrm{C}_{9}$ & $\mathrm{Do}_{9}$ & 8372.00 & 8372.00 & 0.00 & 0.0 \\
\hline
\end{tabular}

ously approximated by a given scale. We find a marked minimum at twelve notes, and in order to better this the number of notes must rise to nineteen. Contrary to what one might naively expect, simply raising the number of notes or, equivalently, diminishing the interval between adjacent notes, does not automatically achieve a better approximation to the harmonic intervals. As a consequence, the number of notes of an equal-tempered scale must be determined by this condition and cannot be arbitrarily chosen. In Fig. 5 we can see that the function also has a significant minimum for thirty-four notes, and if we continue the construction of the golden scale one step further we find a scale with thirty-four notes (Table 3). Our golden scale construction, then, provides scales with optimal numbers of notes to best preserve the harmonic intervals.

In axiomatic terms, the construction of the order $n$ scale from the order $n-1$ scale can be summarized thus: first, include the next convergent of the golden section, $c_{n}$. Construct the geometric mean of the interval $\left(c_{n-1}, c_{n}\right)$ and its reflections in the previous geometric mean mirrors. Include all the possible reflections of the convergents obtained up to this point in the geometric mean mirrors, following the rule that an interval may not be greater than $c_{n-2} / c_{n+1}$, nor less than $c_{n} / c_{n-1}$ (these ratios are to be inverted depending on whether $n$ is odd or even, so that they are always greater than one). If an interval remains too large after including all possible rationals, then it must be subdivided by the irrational geometric mean until the rule is satisfied. The completed scale should be palandromic. There is very little that is arbitrary in the construction of these scales: everything comes given by just one number, the golden section. The original notes are convergents of the golden section, the admissible intervals are quotients of convergents of the golden section, and the symmetry axes are geometric means between neighbouring convergents of the golden section. With the exception of the first in the series, the pentatonic golden scale, the golden scales are not just, with all intervals rational, but neither are they equaltempered, with all intervals irrational. As they include both rational and irrational intervals, we may term them mixed scales.

\section{Playing and transposing with golden scales in equal temperament}

As with any other non-equal-tempered scale, the golden scales cause problems for transposition. The golden scale of twelve notes is interesting for compositional purposes since it has the same number of notes as the usual equal-tempered one, while some of the notes deviate appreciably from the corresponding equal-tempered ones. An interesting way to use this scale while maintaining the possibility of transposition is to approximate it by a subset of an equal-tempered scale of a greater number of notes. As the next step in the golden scale construction 
Table 3: The golden scale construction carried out up the the sixth convergent of the golden mean, $c_{6}=$ $8 / 13$, gives us a thirty-four-note golden scale. This contains within it the whole twelve-note golden scale; the additional notes are reflections of convergents of the golden section, plus irrational notes from the inclusion of mirrors at the geometric means of the intervals. It is hence a mixed scale with both rational and irrational intervals. From a musical viewpoint, this allows one to play with consonance, dissonance, and tonality.

\begin{tabular}{|c|c|c|c|c|}
\hline Note & Interval & \multicolumn{2}{|c|}{$\begin{array}{l}\text { Associated Note of } \\
\text { Twelve-Note Scale }\end{array}$} & $\begin{array}{r}\text { Thirty-Four-Note } \\
\text { Golden Scale (Hz) }\end{array}$ \\
\hline 0 & $1 / 2$ & $\mathrm{C}_{8}$ & $\mathrm{Do}_{8}$ & 4186.00 \\
\hline 1 & $20 / 39$ & & & 4293.33 \\
\hline 2 & $25 / 48$ & & & 4360.42 \\
\hline 3 & $8 / 15$ & $C^{\sharp}$ & $\mathrm{Do}^{\sharp}$ & 4465.07 \\
\hline 4 & $13 / 24$ & & & 4534.83 \\
\hline 5 & $5 / 9$ & $\mathrm{D}$ & $\operatorname{Re}$ & 4651.11 \\
\hline 6 & $\sqrt{5} /(3 \sqrt[4]{3})$ & & & 4741.47 \\
\hline 7 & $1 / \sqrt{3}$ & & & 4833.58 \\
\hline 8 & $\sqrt[4]{3} / \sqrt{5}$ & & & 4927.48 \\
\hline 9 & $3 / 5$ & $D^{\#}$ & $\mathrm{Re}^{\#}$ & 5023.20 \\
\hline 10 & $8 / 13$ & & & 5152.00 \\
\hline 11 & $5 / 8$ & E & Mi & 5232.50 \\
\hline 12 & $16 / 25$ & & & 5358.08 \\
\hline 13 & $13 / 20$ & & & 5441.80 \\
\hline 14 & $2 / 3$ & $\mathrm{~F}$ & $\mathrm{Fa}$ & 5581.33 \\
\hline 15 & $\sqrt{2} \sqrt[3]{3} / 3$ & & & 5691.98 \\
\hline 16 & $1 / \sqrt[3]{3}$ & & & 5804.82 \\
\hline 17 & $1 / \sqrt{2}$ & $\mathrm{~F}^{\sharp}$ & $\mathrm{Fa}^{\sharp}$ & 5919.90 \\
\hline 18 & $\sqrt[3]{3} / 2$ & & & 6037.26 \\
\hline 19 & $3 /(2 \sqrt{2} \sqrt[3]{3})$ & & & 6156.94 \\
\hline 20 & $3 / 4$ & G & Sol & 6279.00 \\
\hline 21 & $10 / 13$ & & & 6440.00 \\
\hline 22 & $25 / 32$ & & & 6540.63 \\
\hline 23 & $4 / 5$ & $\mathrm{G}^{\sharp}$ & $\mathrm{Sol}^{\sharp}$ & 6697.60 \\
\hline 24 & $13 / 16$ & & & 6802.25 \\
\hline 25 & $5 / 6$ & A & $\mathrm{La}$ & 6976.67 \\
\hline 26 & $\sqrt{5} /(2 \sqrt[4]{3})$ & & & 7112.20 \\
\hline 27 & $\sqrt{3} / 2$ & & & 7250.36 \\
\hline 28 & $3 \sqrt[4]{3} /(2 \sqrt{5})$ & & & 7391.21 \\
\hline 29 & $9 / 10$ & $\mathrm{~A}^{\sharp}$ & $\mathrm{La}^{\sharp}$ & 7534.80 \\
\hline 30 & $12 / 13$ & & & 7728.00 \\
\hline 31 & $15 / 16$ & B & $\mathrm{Si}$ & 7848.75 \\
\hline 32 & $24 / 25$ & & & 8037.12 \\
\hline 33 & $39 / 40$ & & & 8162.70 \\
\hline 34 & $1 / 1$ & $\mathrm{C}_{9}$ & $\mathrm{Do}_{9}$ & 8372.00 \\
\hline
\end{tabular}

gives a thirty-four-note scale, and because this scale contains all the intervals of the twelve-note one, we can approximate the notes of the latter with the notes of an equal-tempered scale of thirty-four steps. As we can see in Fig. 5, this choice is a better approximation to the harmonic intervals, in the sense of having a smaller mean quadratic dispersion. In Table 4 we show the notes of the thirty-four note equal-tempered scale that approximate the corresponding notes in the twelve-note golden scale, and the differences between them expressed in cents. The maximum deviation is of the order of six cents, very near to the just noticeable difference limen. Thus, 


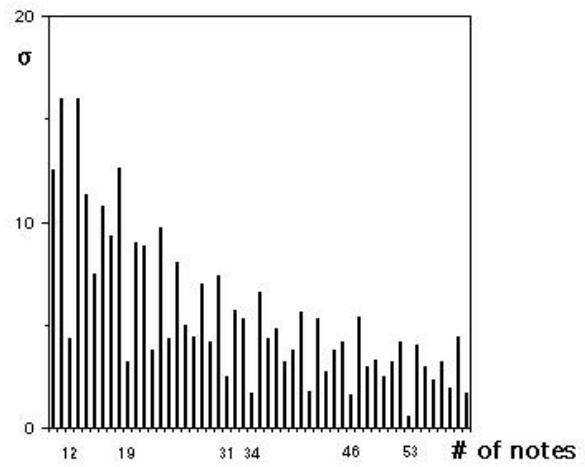

Figure 5: Mean quadratic dispersion $\sigma$ as a function of the number of notes in an equal-tempered scale. This number is the square of the difference between the note of the equal-tempered scale that best approximates each harmonic interval, multiplied by the relative weight of each interval and summed over all the intervals. The weights of the intervals are set such that the fifth weighs more than the fourth, which weighs more than the major third and major sixth, which weigh more than the minor third and minor sixth. $\sigma$ is then an indication of the degree to which a given equal-tempered scale approximates all the harmonic intervals of Table 1. There is a marked minimum for the usual twelve-note scale which coincides with the number of notes of the golden scale (the fifth convergent of the golden number). To obtain a better value, the number of notes must rise to nineteen. The two following minima are at thirty-one and thirty-four notes, and the latter value coincides with the number of notes of the golden scale developed until the sixth convergent of the golden number, 8/13.

the thirty-four-note equal-tempered scale can be used as a very good approximation to the golden one, with the benefit that in the equal-tempered scale a musical composition can be transposed without difficulty. Moreover, we can change tonality within microtonal intervals, by going to a non-twelve interval available in the thirty-fournote scale. This is a general principle: we can play an order $n-1$ golden scale as a subset of the order $n$ one and, for sufficiently high $n$ the order $n$ scale can be approximated by an equal-tempered one with the same number of notes. Thus, the order $n-1$ scale can be played with transposition in this latter scale with the additional possibility of microtonal change of tonality. The example above demonstrates that, for practical applications, it is not necessary to raise further the number of notes; the thirty-four-note scale $(n=6)$ is at the threshold of our sensorial pitch sensitivity for a just generation of all the harmonic intervals that we have considered relevant to the construction of a useful musical scale.

\section{Can our senses be viewed as generic nonlinear systems?}

We have shown that we can construct meaningful musical scales based solely on number-theoretical properties of the continued fraction development of the golden number and its convergents. But is the rôle of the golden number in musical aesthetics a coincidence; is there some connection with the rôle played by the same number in visual aesthetics?

The development of dynamical systems theory is changing our view about nonlinear phenomena in nature. We have mentioned the cultural hypothesis which considers that the rôle of the golden number in aesthetics is due to the ubiquity of this number in natural phenomena. It is now clear that in many cases this rôle in natural phenomena is due to underlying dynamical mechanisms (Ball, 1998). Number theory in general, and certain numbers such as the golden number in particular, play important parts in the dynamics of nonlinear systems (González \& Piro, 1983; Cartwright et al., 1999b). To give just one example, patterns seen in phyllotaxis and in the generation of Fibonacci spirals have been reproduced in a dynamics experiment on the organization of ferrofluid drops in a silicone oil (Douady \& Couder, 1992). 
Table 4: The notes of the thirty-four-note equal-tempered scale that approximate the corresponding notes in the twelve-note golden scale, and the differences between them expressed in cents.

\begin{tabular}{llrr}
\hline $\begin{array}{r}\text { Twelve-Note } \\
\text { Golden Scale }\end{array}$ & $\begin{array}{r}\text { Thirty-Four Note } \\
\text { Equal-Tempered Scale }\end{array}$ & $\begin{array}{r}\text { Difference } \\
\text { (cents) }\end{array}$ \\
\hline $\mathrm{C}$ & $\mathrm{Do}$ & 0 & 0 \\
$\mathrm{C}^{\sharp}$ & $\mathrm{Do}^{\sharp}$ & 3 & 5 \\
$\mathrm{D}$ & $\mathrm{Re}$ & 5 & 6 \\
$\mathrm{D}^{\sharp}$ & $\mathrm{Re}^{\sharp}$ & 9 & -2 \\
$\mathrm{E}$ & $\mathrm{Mi}$ & 11 & -2 \\
$\mathrm{~F}$ & $\mathrm{Fa}$ & 14 & 4 \\
$\mathrm{~F}^{\sharp}$ & $\mathrm{Fa}^{\sharp}$ & 17 & 0 \\
$\mathrm{G}$ & $\mathrm{Sol}$ & 20 & -4 \\
$\mathrm{G}^{\sharp}$ & $\mathrm{Sol}$ & 23 & 2 \\
$\mathrm{~A}$ & $\mathrm{La}$ & 25 & 2 \\
$\mathrm{~A}^{\sharp}$ & $\mathrm{La}$ & 29 & -6 \\
$\mathrm{~B}$ & $\mathrm{Si}$ & 31 & -5 \\
$\mathrm{C}$ & $\mathrm{Do}$ & 34 & 0 \\
\hline
\end{tabular}

Musical scales are constructed around musical intervals, which may be consonant or dissonant (Table 1). Here we must be careful to distinguish the concept of musical consonance from that of psychoacoustical consonance (Plomp \& Levelt, 1965); psychoacoustical consonance makes use of the idea of roughness, but many observations about the consonance of musical intervals cannot be explained on those grounds. The first to put forward an explanation for musical consonance was Rameau (Rameau, 1722). In his theory of harmony, Rameau assumed that musical chords conveyed information about a fundamental sound: a bass note representing the tonal meaning of the chord. Related ideas are Rieman's aural subharmonics (Rieman, 1903), and those that have their origins in Tartini's third tone (Tartini, 1754). More recently, Terhardt gave fresh impetus to the theory of fundamental bass, proposing that the psychoacoustical phenomenon of virtual or residue pitch may be ascribed to it (Terhardt, 1974). However, Terhardt's ideas lack a clear connection between the physical parameters of the sound and the virtual pitch response. Except for von Helmholtz's ideas on virtual pitch (von Helmholtz, 1863), which make use of combination tones, other theories of the phenomenon show the same lack of physical significance. Recently we proposed a new theory of residue perception, based on nonlinear dynamics (Cartwright et al., 1999a, 2001). Following the line of reasoning of Terhardt, this becomes ipso facto a physical explanation for musical consonance. Our theory is based on a type of dynamical attractor termed a three-frequency resonance. These resonances are hierarchically organized following rules borrowed from number theory and confirmed through simulation and experiment (Cartwright et al., 1999b). In this hierarchical ordering, a central part is played by the generalization of a number-theoretical operation known as the Farey sum, which also plays a central rôle in the organization of synchronized responses in periodically forced oscillators (González \& Piro, 1983). There, the Farey sum leads to a privileged rôle for the golden section.

We propose that following our theory of residue perception, musical consonance may be explained in physical terms. The auditory system is a very complex and highly nonlinear dynamical system, so we expect that universal dynamical attractors may convey a perceptual and functional meaning in neural processing. Universal dynamical attractors of interest for pitch perception, that is three-frequency resonances, are organized by means of a number-theoretical operation, the generalized Farey sum, which implies a privileged rôle for the golden section in their hierarchical organization. The part the golden section plays in the hierarchical organization of musical intervals, outlined in this paper, may then be a consequence of the dynamical ordering pointed out above at the level of neural processing in the auditory system. A final hypothesis can be proposed: the tonal meaning and the relative consonance of a musical chord may be described by the stability of a dynamical attractor which represents the residue pitch. This idea is quantitatively testable, because this stability can be measured through different dynamical indicators. 
Our theory for the pitch perception of complex sounds by the human auditory system demonstrates that the auditory system's response to musical sounds is compatible with the universal response of a nonlinear dynamical system to such stimuli. Because neuronal networks are very complex dynamical systems, this is not such an unexpected result. It may be on this basis that the presence of the golden number in musical aesthetics can be explained: harmonic intervals are another manifestation of the universal nonlinear behaviour associated with pitch perception. The same phenomena may occur at the level of the visual system, because object identification appears to correspond physiologically to synchronization of neuron populations to a given frequency (Engel et $a l .$, 1992). The presence of different elements in an image might be then detected through different neuronal groups that synchronize to different frequencies. And this returns us to the premise of our theory: the nonlinear interaction of two or more frequencies produces resonances that are hierarchically arranged in a manner described by the golden mean. Thus, the more recent results take us nearer to the more ancient theories, to the Pythagorean dogma that all the universe is described by numbers and rhythms (Allott, 1994) — in modern terms number theory and dynamics - and that nature is from all points of view similar to itself — in modern terms universality. We may conclude with the words of the Gothic architect Jean Vignot on the continuation of the work on Milan cathedral in 1392: "Ars sine scientia nihil est".

\section{Bibliography}

Allott, R. [1994] “The Pythagorean perspective: The arts and sociobiology,” J. Social and Evolutionary Syst. 17, 71-90.

Ball, P. [1998] The Self-Made Tapestry: Pattern Formation in Nature (Oxford University Press).

Cartwright, J. H. E., González, D. L. \& Piro, O. [1999a] “Nonlinear dynamics of the perceived pitch of complex sounds," Phys. Rev. Lett. 82, 5389-5392.

Cartwright, J. H. E., González, D. L. \& Piro, O. [1999b] “Universality in three-frequency resonances,” Phys. Rev. E 59, 2902-2906.

Cartwright, J. H. E., González, D. L. \& Piro, O. [2001] "Pitch perception: A dynamical-systems perspective," Proc. Natl Acad. Sci. USA 98, 4855-4859.

Douady, S. \& Couder, Y. [1992] "Phyllotaxis as a physical self-organized growth process," Phys. Rev. Lett. 68, $2098-2101$.

Engel, A. K., König, P., Kreiter, A. K., Schillen, T. B. \& Singer, W. [1992] “Temporal coding in the visual cortex: New vistas on integration in the nervous system," Trends in Neurosciences 15, 218-226.

Ghyka, M. C. [1977] The Geometry of Art and Life (Dover).

González, D. L. \& Piro, O. [1983] "Chaos in a nonlinear driven oscillator with exact solution,” Phys. Rev. Lett. 50, 870-872.

Hardy, G. H. \& Wright, E. M. [1975] An Introduction to the Theory of Numbers (Oxford University Press) fourth edition.

Herz-Fischler, R. [1998] A Mathematical History of the Golden Number (Dover).

Huntley, H. E. [1970] The Divine Proportion: A Study In Mathematical Beauty (Dover).

James, J. [1993] The Music of the Spheres (Grove Press).

Lendvai, E. [1966] "Duality and synthesis in the music of Béla Bartók," in Kepes, G., editor, Module, Proportion, Symmetry, Rhythm (George Braziller).

Newton, I. [1952] Opticks (Dover).

Pacioli, L. [2001] Divine Proportion (Arabis Books).

Plomp, R. \& Levelt, W. [1965] “Tonal consonance and critical bandwidth,” J. Acoust. Soc. Am. 38, 548-560.

Rameau, J. P. [1722] Traité de l'harmonie (Paris).

Rieman, H. [1903] Catechism of Orchestration (London).

Schroeder, M. R. [1990] Number Theory in Science and Communication (Springer). 
Schroeder, M. R. [1992] Fractals, Chaos, Power Laws: Minutes from an Infinite Paradise (W. H. Freeman).

Tartini, G. [1754] Trattato di Musica (Padua).

Terhardt, E. [1974] “Pitch, consonance, and harmony,” J. Acoust. Soc. Am. 55, 1061-1069.

von Helmholtz, H. L. F. [1863] Die Lehre von dem Tonempfindungen als physiologische Grundlage für die Theorie der Musik (Braunschweig).

Zeki, S. [1999] Inner Vision (Oxford University Press). 